\title{
Design And Characteristic Research of Contact Probe For High-Precision 3D Thread-Measuring Machine
}

Jing YU ( $\nabla$ yujing88@cjlu.edu.cn )

China Jiliang University

Xi-meng Lin

China Jiliang University

Yan-ling Yang

China Jiliang University

Jin-hui Cai

China Jiliang University

\section{Research Article}

Keywords: Contact scanning probe, micro-force probe model, Structural optimization, Static and dynamic characteristics

Posted Date: August 18th, 2021

DOI: https://doi.org/10.21203/rs.3.rs-800819/v1

License: (c) (i) This work is licensed under a Creative Commons Attribution 4.0 International License. Read Full License 


\title{
Design and characteristic research of contact probe for high-precision 3D thread- measuring machine
}

\author{
Jing Yu*, Xi-meng Lin, Yan-ling Yang, Jin-hui Cai \\ College of Metrology and Measurement Engineering, China Jiliang University, Hangzhou, Zhejiang 310018, China
}

\begin{abstract}
A probe is an important part of high-precision thread-measuring machines. Probe design affects the ability of the machine to achieve precision. The development of three-dimensional (3D) thread-measuring machines has mandated strict requirements for matching measuring heads. A contact scanning probe offers the advantages of a relatively high measurement accuracy and stable performance. In this study, the structure of the scanning probe was designed. First, a micro-force probe model was established according to 3D thread characteristics. The "T"-shaped, ballpoint-pen shaped needle was selected according to the characteristics of the detection hole and thread. Thereafter, the dimension parameters of the measuring ball, measuring bar, parallel spring plates, and other components were designed to enable the measuring head to meet the precision requirements. The flexural deformation of the measuring rod was analyzed to determine the appropriate length and diameter of the measuring rod. Finally, the effective static and dynamic characteristics of the head were demonstrated through finite element simulation and experimental measurements. In addition, the static characteristics of the probe were measured. The return error was $0.29 \mu \mathrm{m}$, and the repeatability error was $0.24 \mu \mathrm{m}$. The dynamic characteristics were tested using the percussion method, and the natural frequency was $180 \mathrm{~Hz}$. These results help ensure the precision of the probe and improve the measuring precision of the machine.
\end{abstract}

Keywords: Contact scanning probe; micro-force probe model; Structural optimization; Static and dynamic characteristics.

\footnotetext{
${ }^{*}$ Corresponding author: College of Metrology and Measurement Engineering, China Jiliang University, Hangzhou, Zhejiang 310018, China

Tel.: +8613735546719 .

E-mail address: yujing88@,cjlu.edu.cn (J. Yu).
} 


\section{Introduction}

With rapid developments in science and technology, traditional standards and twodimensional measurement methods must be reevaluated to describe three-dimensional (3D) topographical threads. It is challenging to identify local, elliptic-type [1-4], thintooth problems. Thus, it is difficult to accurately control the assembly quality, jeopardizing the precision of thread-testing machines, which must follow the advanced manufacturing screw-geometry parameters. Therefore, an urgent requirement exists for comprehensive measurements and 3D measurement techniques in the field of screw geometry measurement [5, 6]. Gong [7] studied 3D measurement technology for several years, and in 2015 proposed a high-resolution and high-speed 3D shape measurement technology. The measurement of internal threads differs from that of external threads. A new method using a scanning optical fiber endoscope and an axial stereo vision algorithm was proposed to measure tiny internal 3D surfaces [8]. In 2017, Wakayama T, Takahashi Y, Ono Y et al. proposed a 3D optical measurement method based on machine vision, which was used to measure the $3 \mathrm{D}$ contours of complex and tiny inner surfaces, such as internally threaded holes [9].

As it is particularly important to obtain data regarding the screw surface, the probe is the key precision component of a $3 \mathrm{D}$ screw-measuring machine, and its structure restricts the measuring precision of the entire machine. Probes can be classified as contact or optical probes according to the measurement method [10-12]. Contact probes offer a high measurement accuracy and stable performance and are used extensively in 3D measurement. The design of contact probes by universities and research institutes is varied. $\mathrm{Li}$ introduced the principle and uncertainty of the measurement of the Wiener probe, including the capacitive, piezoresistive, inductive, imaging, photoelectric, and resonant types, and its resolution was found to be between 1 and $100 \mathrm{~nm}$ [13].

Researchers have used finite element software to simulate and study the mechanical and structural properties of probes, such as needle stiffness and structural analysis [1416], with the aim of obtaining the optimal geometric design [17, 18]. The finite element analysis technique has also been verified experimentally to obtain a new probe with tiny contact loads [19].

Regarding the measurement of deep thread holes, there is significant work to do to design the most appropriate probe structure. Most of the studies at home and abroad 
focus upon the combination of big ball and fine needle. Given that the probe structure design for deep threaded holes is still lacking, it is necessary to develop a more specialized contact probe for scanning the grooves of thread. Thus, it is necessary to develop a special contact probe for scanning any depth of threaded grooves. In this study, a novel, high-precision contact scanning probe with a force-measuring control on the $\mathrm{mN}$ level was designed. The probe model was established based on the 3D characteristics of screw threads. The geometric dimensions of the measuring ball, measuring rod, and parallel reed were determined to meet the requirements of a highprecision measurement according to Hertz's law and the dynamic model. Moreover, a finite element analysis was conducted to determine the static and dynamic characteristics (natural frequency) of the model.

To further demonstrate the feasibility of the proposed model and simulation, the probe was tested by experiments, and the results are provided in this paper. By combining the finite element simulation results and experimental data, the static and dynamic characteristics of the contact scanning probe were proven. The proposed method can realize stable and reliable 3D contact scanning measurements for the thread profile.

\section{Probe model}

The contact probe is divided into three parts: the ball-needle, rod, and elastic mechanism. Various measuring ball shapes are available for different threaded objects and complex measuring paths. The common structure is a combination of a large measuring ball and measuring needle; that is, the diameter of the measuring ball is larger than that of the measuring needle, as illustrated in Fig. 1. In terms of the contact force measurement, a larger ball and shorter rod length result in a higher measurement accuracy. However, for the probe hole and threaded characteristics, the "T"-type, ballpoint-pen shape should be selected [12], as demonstrated in Fig. 2, to improve the uphill and downhill in scanning process problems, such as damage caused by the needle on the surface and the needle being stuck. Considering the depth of the measured thread and measuring principle of the measuring machine, a measuring rod extension is incorporated to meet the measurement requirements. 


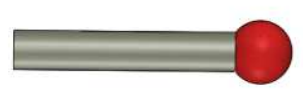

Fig. 1 Common ball-needle structure

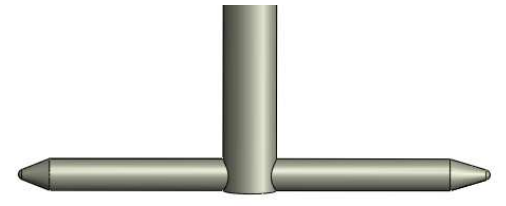

Fig. 2 Structure of special probe with "T"-type, ballpoint-pen shape for thread measurement.

The elastic mechanism primarily consists of three parts: an elastic element, a fixed body, and a connecting body. The elastic element is the key induction component of the structure, which determines the probe measurement performance. To ensure sensitivity, parallel spring plates are used as the micro-guide rail. This mechanism provides high guiding precision and is easy to process. The fixed and connecting bodies should be constructed using materials with low elasticity and light weight, such as titanium and aluminum alloys. The overall design is presented in Fig. 3. Two pairs of parallel spring sets are nested to make the entire structure compact. The load-bearing motion state and working principle of the probe under ideal conditions are depicted in Fig. 4. When the probe is under transverse stress, the left and right parallel springs are stretched, and the measuring ball and measuring bar drive the translational plate to perform a transverse rigid displacement. When a longitudinal force is exerted, the upper and lower parallel springs are stretched, and the measuring ball and measuring bar drive the translational plate to create a rigid longitudinal displacement. The probe of the $3 \mathrm{D}$ thread measuring machine is not only used for positioning but also for measuring. The probe adopts the principle of micro-force control, and a grating system with high precision is installed to measure the relative displacement of the translational and fixed plates. It cooperates with the $3 \mathrm{D}$ thread measuring machine to realize the feedback structure with a high precision and stable force value. 

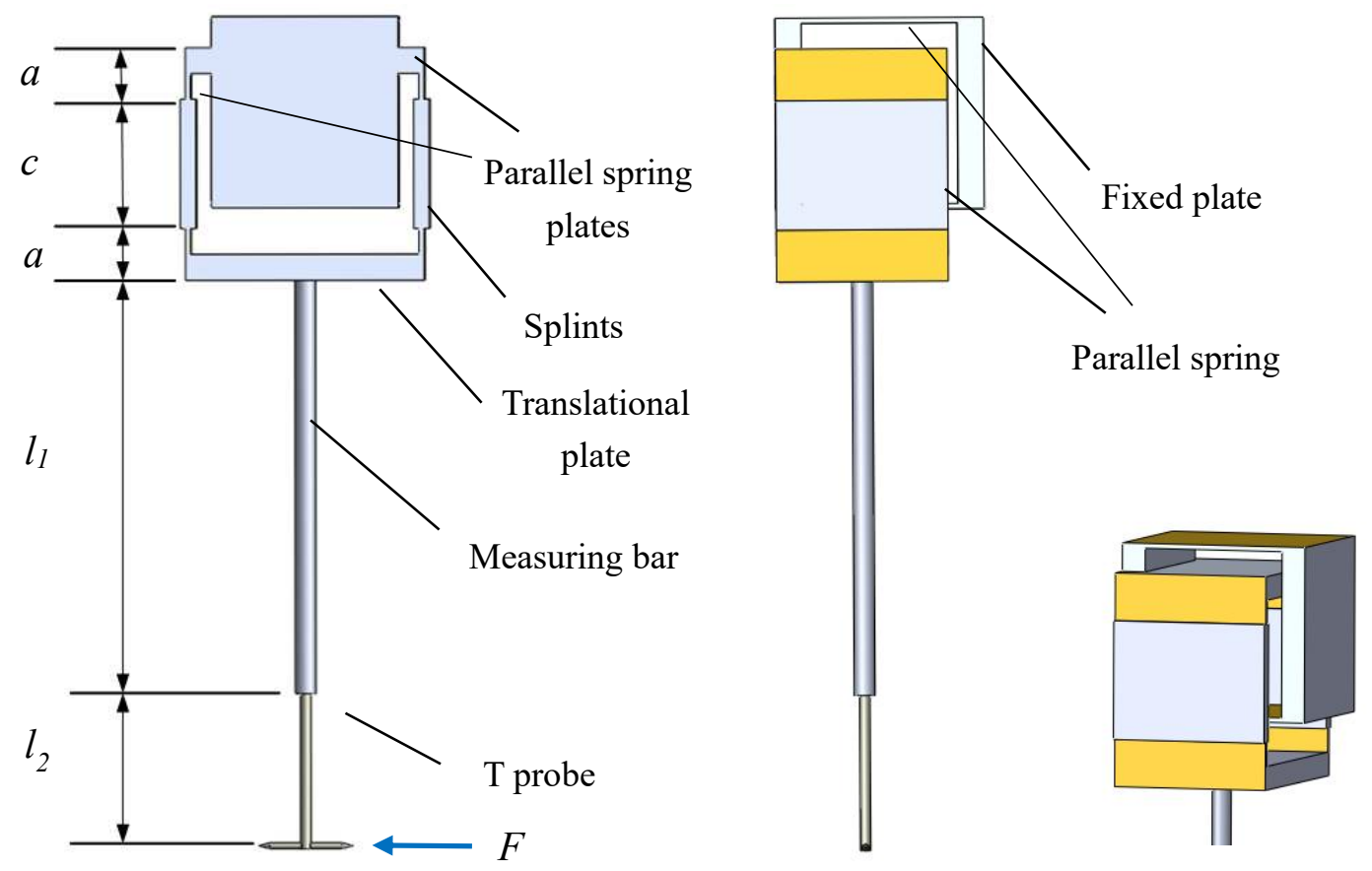

Fig. 3 Contact probe structure of 3D thread-measuring machine

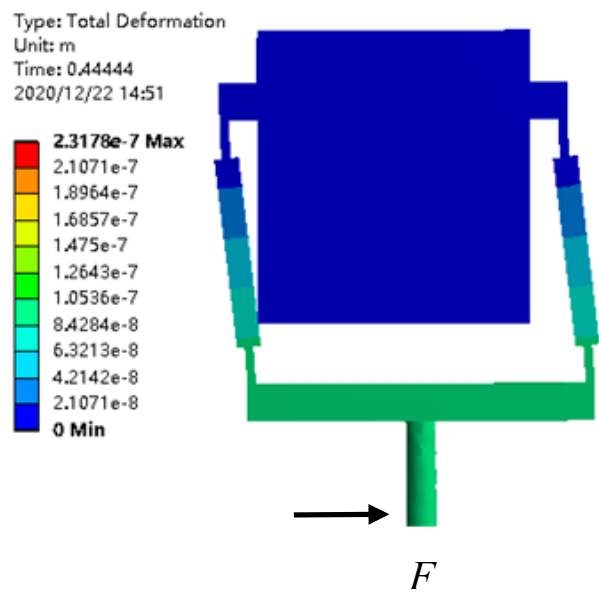

(a) Transverse forces

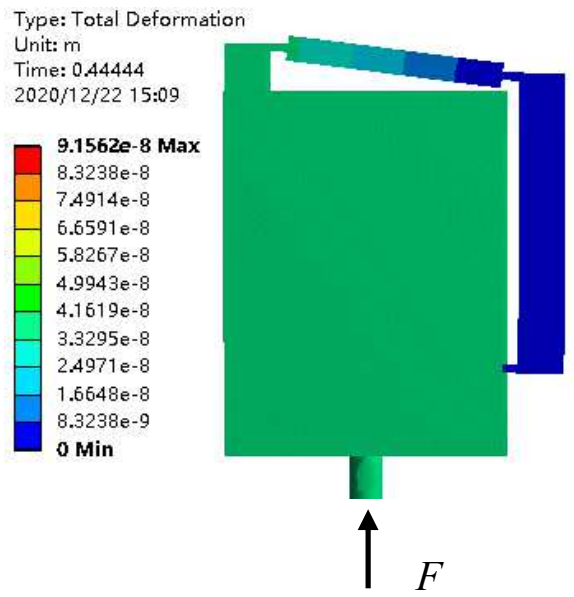

(b) Longitudinal forces

Fig. 4 Theoretical motion state of the probe

\section{Design of geometric parameters}

The measurement precision is determined by the geometric parameters of the probe system, including the measuring ball radius; measuring bar length and diameter; the parallel spring plate thickness, length, and width; and the splint length. 


\subsection{Design of measuring ball radius}

In micro- or nano-scale measurement, considering the influence of the stylus tip radius on the measurement error for a complex thread scanning path, the ball should be of the minimum possible size; however, it should still meet the requirements of the measurement force. According to Hertz's law, the contact force and probe radius relationship can be used to calculate the forced deformation of the ball in contact with the thread being measured, as illustrated in Fig. 5. The radius of the contact surface $c$ is determined as follows:

$$
c=\sqrt[3]{\frac{3 r}{4}\left(\frac{1-u_{1}^{2}}{E_{1}}+\frac{1-u_{2}^{2}}{E_{2}}\right) F},
$$

where $r$ is the ball radius, $F$ is the contact force, $u_{1}$ and $u_{2}$ are the Poisson's ratios of the two contact materials; $E_{1}$ and $E_{2}$ are the elastic moduli of the two contact materials.

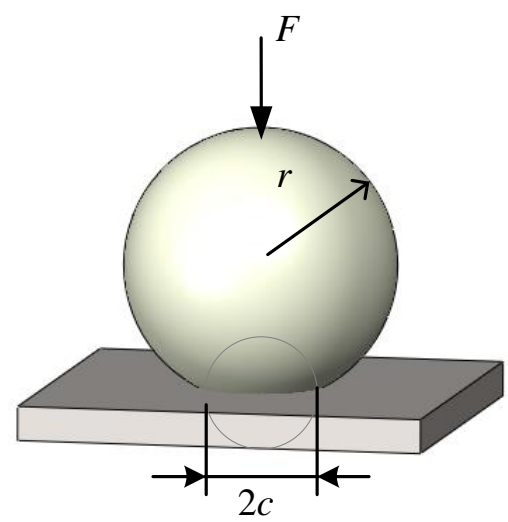

Fig. 5 Ball surface contact deformation under force

The contact stress is calculated as follows:

$$
\sigma=\frac{3 F}{2 \pi c^{2}}=\frac{1}{\pi} \sqrt[3]{6 F\left(\frac{\frac{1}{r}}{\frac{1-u_{1}^{2}}{E_{1}}+\frac{1-u_{2}^{2}}{E_{2}}}\right)^{2}} .
$$

Equation (2) demonstrates that the probe contact stress is related to the measuring ball radius, measuring ball material, and measured thread material. To protect the needle, the stress yield limit of the measuring ball should be slightly larger than the measured thread. Considering the cost, tungsten carbide should be selected as the measuring ball, with the following parameters: elastic modulus: $450 \mathrm{GPa}$, Poisson's ratio: 0.24, and stress yield limit: $800 \mathrm{MPa}$. The measured force is $F=100 \mathrm{mN}$, and this is substituted into Equation (2) to obtain the following relationship: 


$$
\sigma=7.4 r^{-\frac{2}{3}}
$$

The maximum contact stress should be less than the stress yield limit of the measured thread; considering the material that is, $\sigma_{\max }<500 \mathrm{MPa}$, and the equation is solved to obtain $r>0.0018 \mathrm{~mm}$. Considering the processing technology and the service life of the probe, we chose the ball radius of this structure to be $0.02 \mathrm{~mm}$ for the measurement.

\subsection{Measuring bar design}

The ratio of the diameter $d$ to the length $l$ of the measuring rod affects the deflection induced by the measuring force. A larger ratio results in smaller deformation and higher measurement accuracy. The rod deformation error can be obtained through a mechanical analysis, as illustrated in Fig. 3. Ignoring the weight of each part, $a, b, h$, $E_{0}$, and $J_{0}$ denote the parallel spring length, width, thickness, elastic modulus, and moment of inertia, respectively; $l_{1}, d_{1}, E_{1}$, and $J_{l}$ represent the rod length, diameter, elastic modulus, and moment of inertia, respectively; $l_{2}, d_{2}, E_{2}$, and $J_{2}$ denote the needle length, diameter, elastic modulus, and moment of inertia, respectively. Moreover, $c$ is the length of the parallel spring splint. When measuring the thread, $F$ is applied to the measuring end, and the displacement between the measuring ball and translational plate can be calculated using the energy method.

$$
\begin{gathered}
\delta_{\text {в }}=\frac{F a}{12 E_{0} J_{0}}\left(4 a^{2}+6 a c+3 c^{2}\right)+\frac{F l_{1}^{3}}{3 E_{1} J_{1}}+\frac{F l_{2}^{3}}{3 E_{2} J_{2}} \\
\delta_{\mathrm{T}}=\frac{F a}{12 E_{0} J_{0}}\left(4 a^{2}+6 a c+3 c^{2}\right)
\end{gathered}
$$

The deformation error of the measuring rod is

$$
\delta_{M}=\delta_{B}-\delta_{T}=\frac{F l_{1}^{3}}{3 E_{1} J_{1}}+\frac{F l_{2}^{3}}{3 E_{2} J_{2}}=k \delta_{f} .
$$

Subsequently,

$$
k=\left(\frac{l_{1}^{3}}{E_{1} J_{1}}+\frac{l_{2}^{3}}{E_{2} J_{2}}\right) \frac{4 E 0 J 0}{4 a^{3}+6 a^{2} c+3 a c^{2}} .
$$

The calibration of the probe during measurement constitutes a modification of $k$. The deformation error is related to the elastic modulus, diameter, length, and contact force of the needle and rod. The needle material is similar to the ball's; that is, it has a high 
density and high stiffness. $l_{2}$ should be sufficiently large such that the needle size can be determined according to the ball. However, as the extension rod of the measuring rod plays the role of force transmitter, the mass should not be excessively large, and materials with low density and high stiffness should be selected, such as carbon fibers. Therefore, the length/diameter ratio of the measuring rod extension rod is the determining factor, and the deformation error should be less than the allowable error. According to the error distribution principle, when the diameter range is approximately between 1 and $5 \mathrm{~mm}$, the length/diameter ratio limit of the measuring rod extension rod is 400 . To ensure the safety factor, the length $l_{1}$ and diameter $d_{1}$ of the extension rod are 100 and $8 \mathrm{~mm}$, respectively.

\subsection{Design of parallel spring plates}

The parallel reeds are an elastic element that determine the probe performance, and the use of relatively thin and wide reeds can enhance measurement accuracy [20]. The geometric dimensions of the parallel spring plates are determined according to the magnitude of the measured force. The maximum measured force of the parallel spring plates is

$$
F_{\text {max }}=\frac{b h^{2} \sigma}{3(2 a+c)},
$$

where $\sigma$ is the allowable stress of the parallel reeds.

Combining Equations (5) and (7) yields

$$
\delta_{\text {max }}=\frac{a b h^{2} \sigma}{36 E_{0} J_{0}(2 a+c)}\left(4 a^{2}+6 a c+3 c^{2}\right) .
$$

As indicated in Fig. 6, beryllium copper is selected as the reed material, with length $a=9 \mathrm{~mm}$, width $b=33 \mathrm{~mm}$, thickness $h=0.25 \mathrm{~mm}$, and splint length $c=27 \mathrm{~mm}$. According to Equation (9), the elastic displacement is approximately $4 \mathrm{~mm}$. The maximum elastic displacement of the parallel reed should be a minimum of five times the measuring range of the probe; that is, the range of the probe can be between 0 and $800 \mu \mathrm{m}$. 


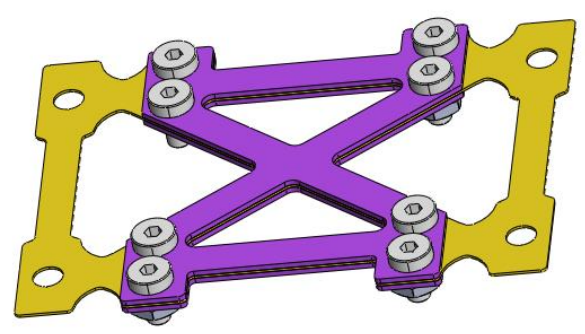

Fig. 6 Reed splint diagram

\section{Finite element analysis of probe}

The geometric dimensions of the measuring ball, measuring bar, parallel spring plates, and splint could be determined according to the design presented in Section 3. Subsequently, the probe performance was analyzed using the finite element method in ANSYS Simulation Software, which can be used for static and dynamic mechanical design analyses. Prior to the simulation, the material of the structure was selected. The parallel reed was made of beryllium copper; the measuring rod was made of carbon fiber; the needle and ball were made of tungsten carbide; the intermediate, fixed, and translational plates were made of aluminum alloy with a high stiffness and low mass.

The static characteristics of the simulated probe included linearity and stiffness; that is, an increasing force was applied to the probe end, the displacements of the probe end and corresponding joints of the translative plate were recorded, and the linearity and stiffness were obtained through fitting calculations. The overall deformation of the probe is depicted in Fig. 7, with a force $F$ applied to the probe end. The red and blue parts in the figure indicate the maximum and zero deformation, respectively. The results of the simulation experiment are presented in Table 1. The stiffness characteristics of the probe was obtained from the displacement and load of the measuring end, whereas the linearity of the probe was obtained from the displacement of the measuring end and translational plate. The data processing is illustrated in Fig. 8. Moreover, the calculated stiffness was $1000 \mathrm{~N} / \mathrm{m}$ with a linearity of $3 \mathrm{~nm}$. In the simulation experiment, the linearity was not influenced by the environment, process error, or other factors, which made the results more ideal. 

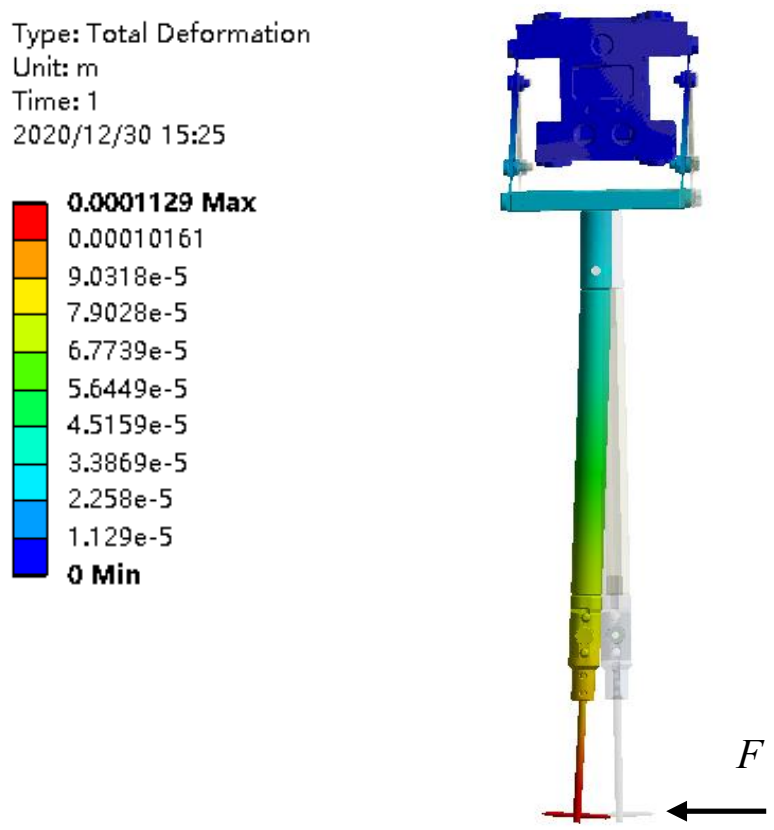

Fig. 7 Simulation of overall probe deformation

Table 1 Results of probe simulation experiment

\begin{tabular}{lllllll}
\hline Test end force load $(\mathrm{mN})$ & 0 & 20 & 40 & 60 & 80 & 100 \\
\hline $\begin{array}{l}\text { Measuring end displacement } 0 \\
(\mu \mathrm{m})\end{array}$ & 20.07 & 40.14 & 60.21 & 80.28 & 100.35 \\
$\begin{array}{l}\text { Displacement of translational } 0 \\
\text { plate }(\mu \mathrm{m})\end{array}$ & 16.72 & 33.45 & 50.18 & 66.90 & 83.625 \\
\hline
\end{tabular}

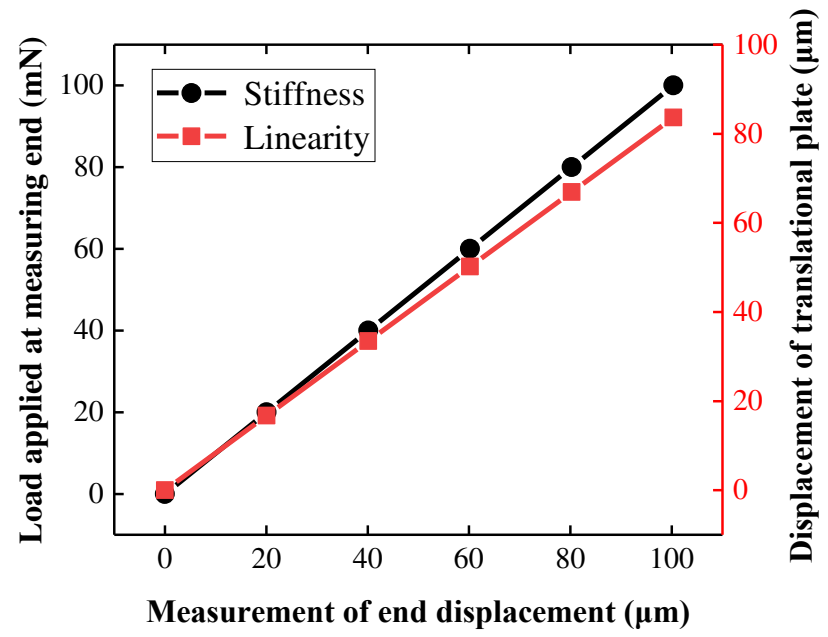

Fig. 8 Static characteristics of probe simulation

The natural frequency of the corresponding order of the model and vibration mode was obtained through the modal analysis of the finite element method. The natural frequency of the transverse translational mode was $193.91 \mathrm{~Hz}$ based on the measured movement direction. According to the dynamic design principle of the mechanical 
structure, a higher natural frequency of the probe, not lower than $100 \mathrm{~Hz}$, is preferable [21]. Therefore, the probe exhibited suitable vibration characteristics.

To explore the influence of the acceleration and weight of the probe on the natural frequency, accelerations of $0.05,0.5$, and $50 \mu \mathrm{m} / \mathrm{s}^{2}$ are selected, and the natural frequency analysis diagram is shown in Fig. 9. It can be seen that the acceleration has little effect on the natural frequency. In the weight simulation, the intermediate materials are varied; aluminum-magnesium alloy, aluminum alloy, and stainless steel are considered. The natural frequency analysis diagram is shown in Fig. 10. It can be seen from Fig. 10 that the natural frequency of the first-order aluminum-magnesium alloy is the largest, and the fifth-order stainless steel is the largest. Therefore, it is evident that the aluminum alloy is generally better.

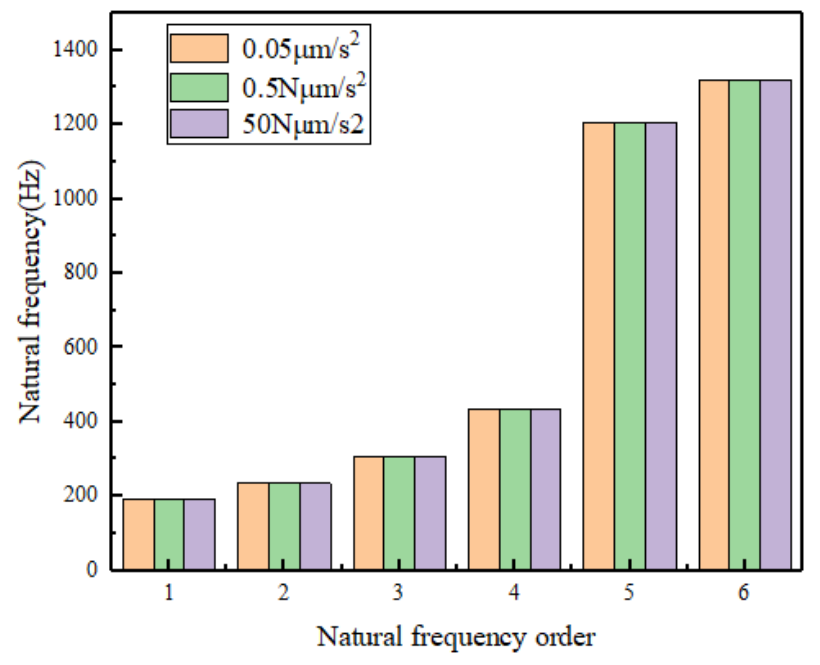

Fig. 9 Effect of acceleration on natural frequency

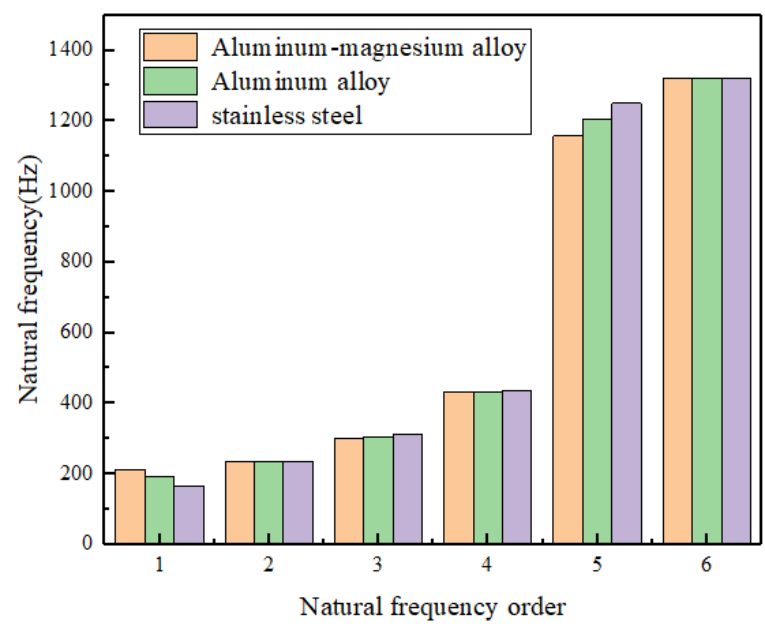

Fig. 10 Influence of weight on natural frequency 
Modal analysis can only reflect the vibration characteristics of the probe itself, but not the dynamic response of the structure during actual work. Therefore, harmonic response analysis of the model was required. The steady-state response of the probe system under a sinusoidal periodic load was obtained through a harmonic response analysis to determine whether the actual working conditions could lead to resonance failure of the probe. The harmonic response analysis is presented in Fig. 11. Within the frequency range of approximately 0 to $1080 \mathrm{~Hz}$, there were three peaks in the measurement direction, and the frequencies were approximately 200, 400, and $1080 \mathrm{~Hz}$. In contrast, the modal analysis was consistent with the natural frequency range of the structure, with three amplitude peaks and a maximum value of $4.87 \mu \mathrm{m}$. Therefore, during the probe measurement, it was necessary to avoid operating frequencies near 200, 400, and $1080 \mathrm{~Hz}$.
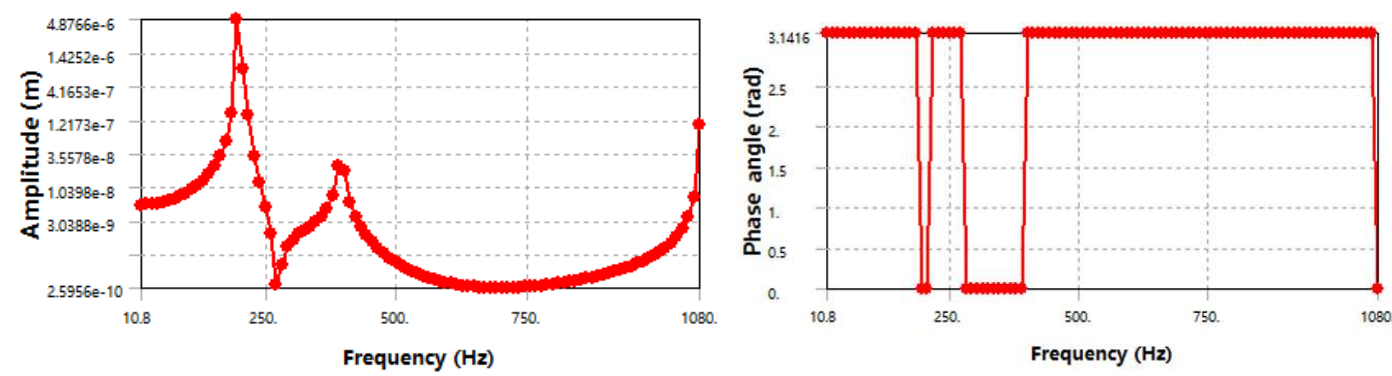

Fig. 11 Amplitude-frequency and phase-frequency characteristics measured by probe

\section{Experimental verification}

\subsection{Static experiment}

The probe was used in conjunction with a thread-measuring machine. The thread was mounted on an electric rotary table. The spatial coordinates of the measured thread were obtained by combining the large displacement of the probe with the R-axis (as indicated in Fig. 12). A small internal displacement was generated when the probe contacted the measured thread. The measurement was performed using the scanning probe control principle. The R-axis represented the lateral movement of the probe and the thread to be tested to establish a direct contact load, whereas the Z-axis along the 
screw generatrix direction represented the longitudinal motion. According to the movement path of the Z-axis, the R-axis received feedback from the microdisplacement generated inside the probe. If the horizontal measuring force exceeded the threshold value, the position of the horizontal motor was adjusted to ensure that the measuring force was within the set value range, the scanning was stopped, and the instrument raised an alarm. The same was true for the vertical measurements.

A high-precision laser interferometer was selected to perform static calibration of the probe. As illustrated in Fig. 12, the probe was first installed on the 3D threadmeasuring machine. Thereafter, the measuring block and linear mirror were fixed on the Y-axis of the 3D thread-measuring machine. The laser and linear interferometers were installed and adjusted so that the beam, spectroscope of the linear interferometer, and linear reflector were on the same horizontal line. The Y-axis was moved to bring the gauge block closer to the measuring ball. After prepressing the probe for $30 \mu \mathrm{m}$, starting from this position, the laser interferometer and Y-axis displacement of the measuring machine and probe displacement data were cleared to zero, and the measuring machine was driven to test the measuring range of the probe with displacements of $50,100,150$, and $200 \mu \mathrm{m}$. The calibrated value was obtained by multiplying the probe displacement shown in Fig. 13 with the linear fitting slope $k_{1}=$ 1.10134, which is the coefficient for static calibration. 


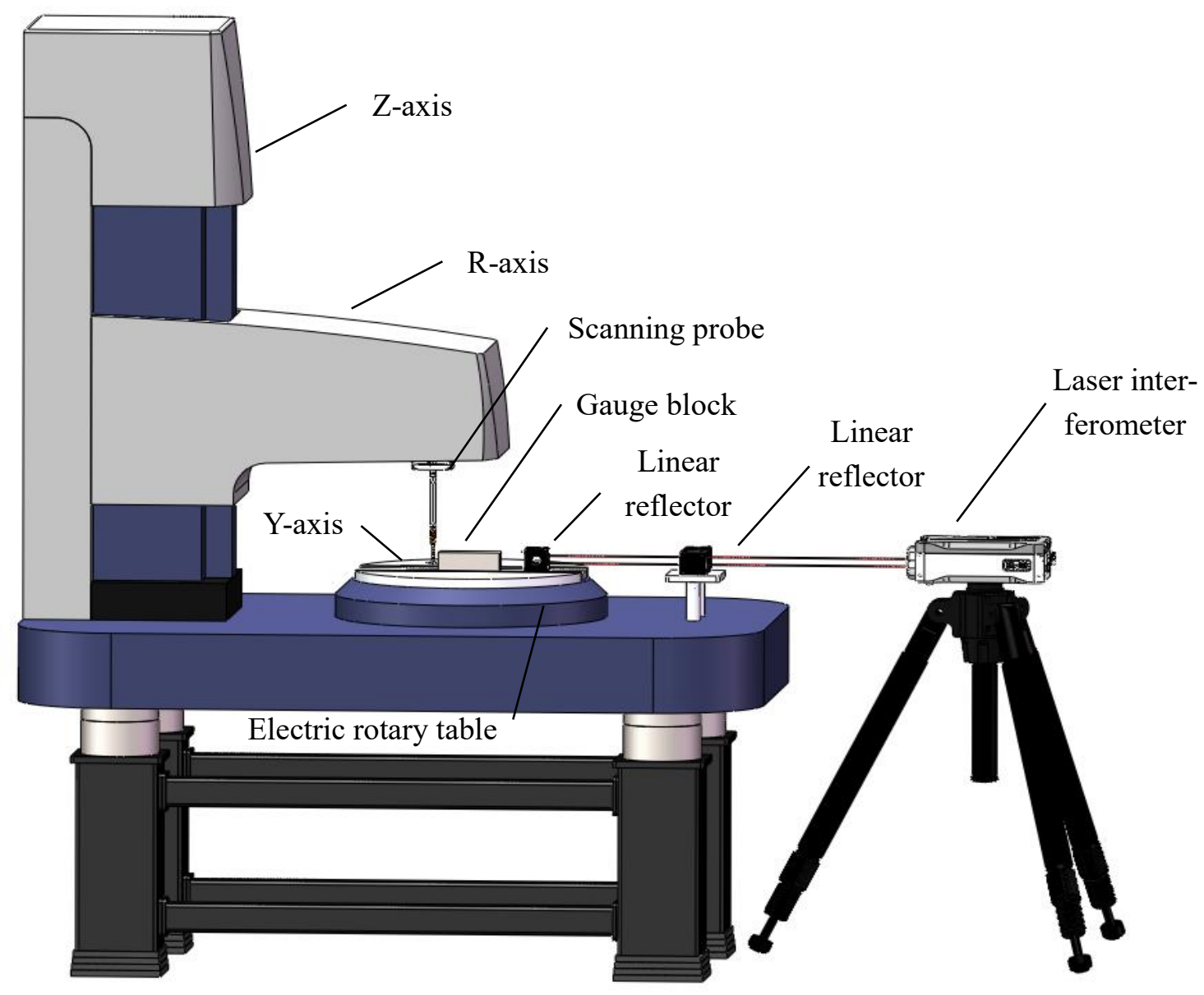

Fig. 12 Apparatus for static experiment of probe

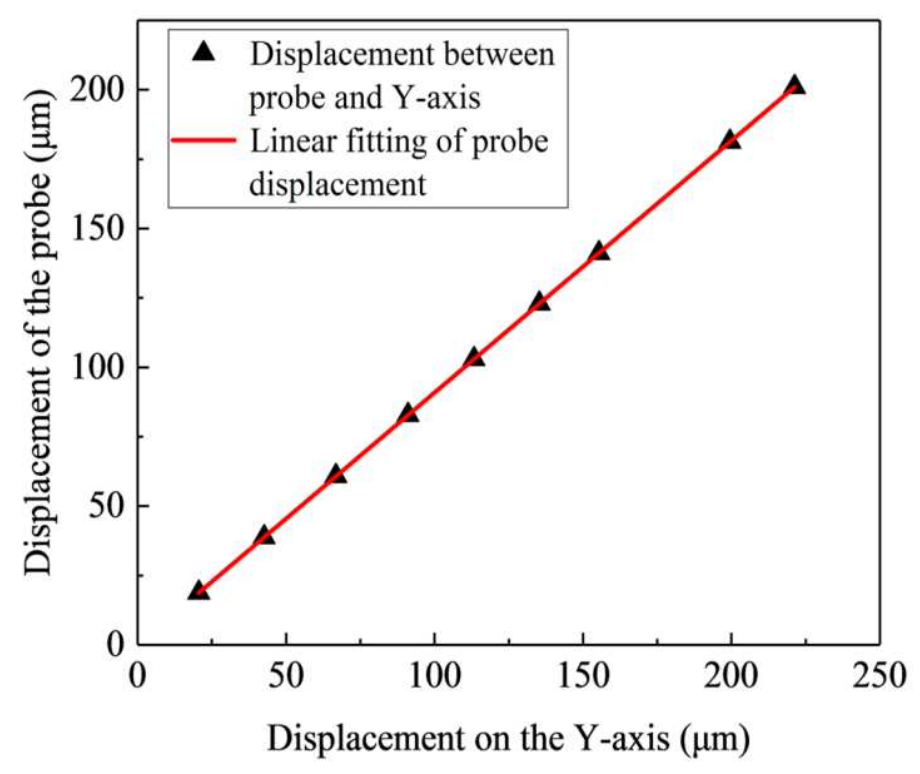

Fig. 13 Probe calibration

The measurement methods for the return trip and repeatability errors were the same as that described above. The measurement process of the return trip error was 
divided into the process and return trips. The repeatability was measured five times, and the data results are presented in Figs. 14 and 15. The test range was 0 to $260 \mu \mathrm{m}$, and the data processing resulted in a return and repeatability error of 0.29 and $0.24 \mu \mathrm{m}$, respectively.

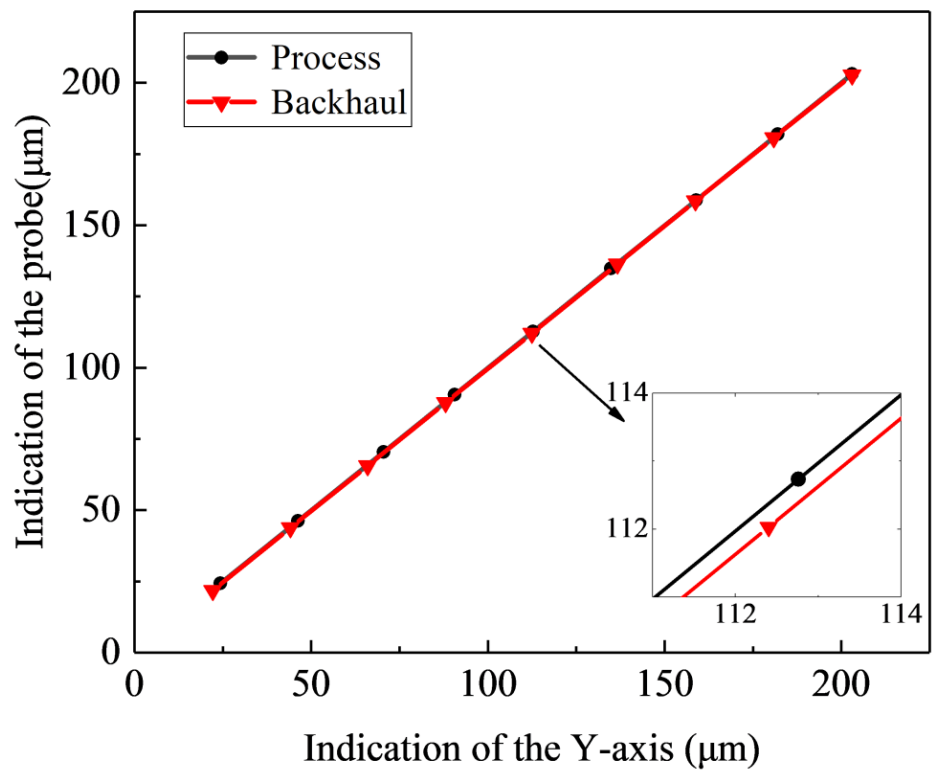

Fig. 14 Return error of probe

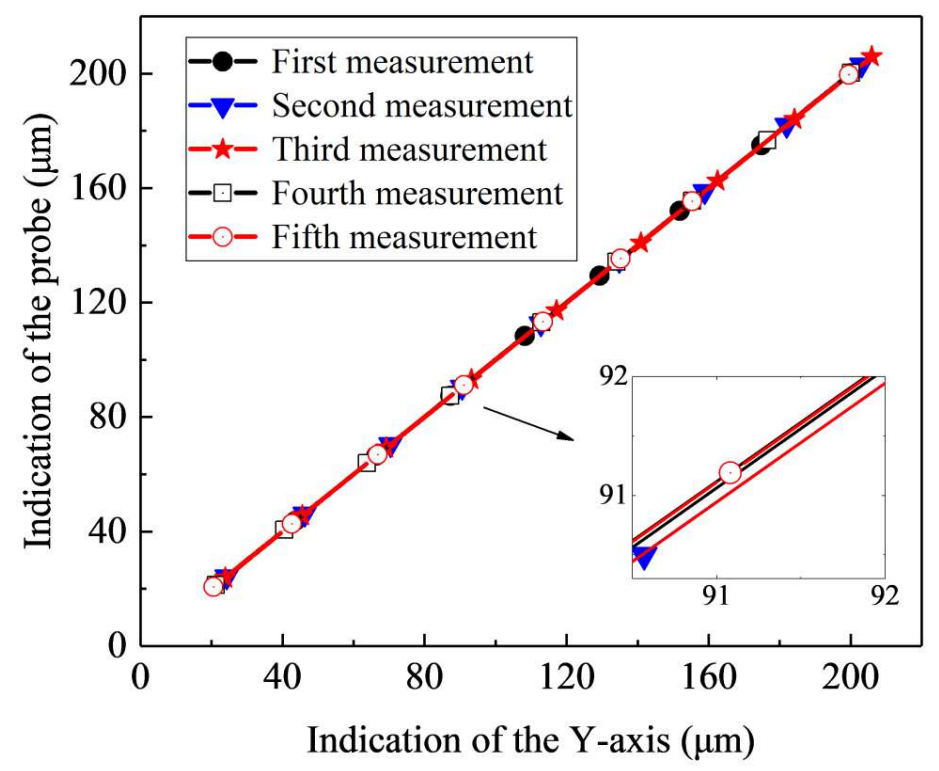

Fig. 15 Repeatability of probe

\subsection{Dynamic experiment}

The dynamic characteristics of the probe, which primarily refer to the natural frequency, play an important role in the scanning process. The natural frequency was 
measured through the free vibration method. The measuring end of the probe system was hammered to cause it to vibrate freely. A high-resolution, $10 \mathrm{~nm}$-long grating was installed inside to record the tiny vibration amplitude of the parallel reed. The waveform of the attenuation vibration of the system and that of the standard time signal were recorded simultaneously. The following relationship between the natural frequency $\omega_{d}$ of the attenuation vibration of the system and the natural frequency $\omega_{f}$ of the probe system exists.

$$
\omega_{f}=\sqrt{\omega_{d}^{2}+\left(\left(\frac{\omega_{d}}{i} \ln \frac{x_{i}}{x_{i+1}}\right) / 2 \pi\right)^{2}},
$$

where $\omega_{d}$ is the vibration frequency; $x_{i}$ and $x_{i+1}$ are the amplitudes in periods $i$ and $i+1$, respectively; $i$ is the number of periods.

The amplitude curve recorded by the percussion method is presented in Fig. 16. The attenuation motion of the probe and the vibration period was $T \approx 0.035 \mathrm{~ms}$, and the vibration frequency was $\omega_{d} \approx 180 \mathrm{~Hz}$. When $i=10$, substituting the value into Equation (10) yielded the calculated natural frequency of the probe as three, which was similar to the simulation results. This demonstrates the reliability of the simulation and the credibility of the natural frequency measurement evaluation of the probe.

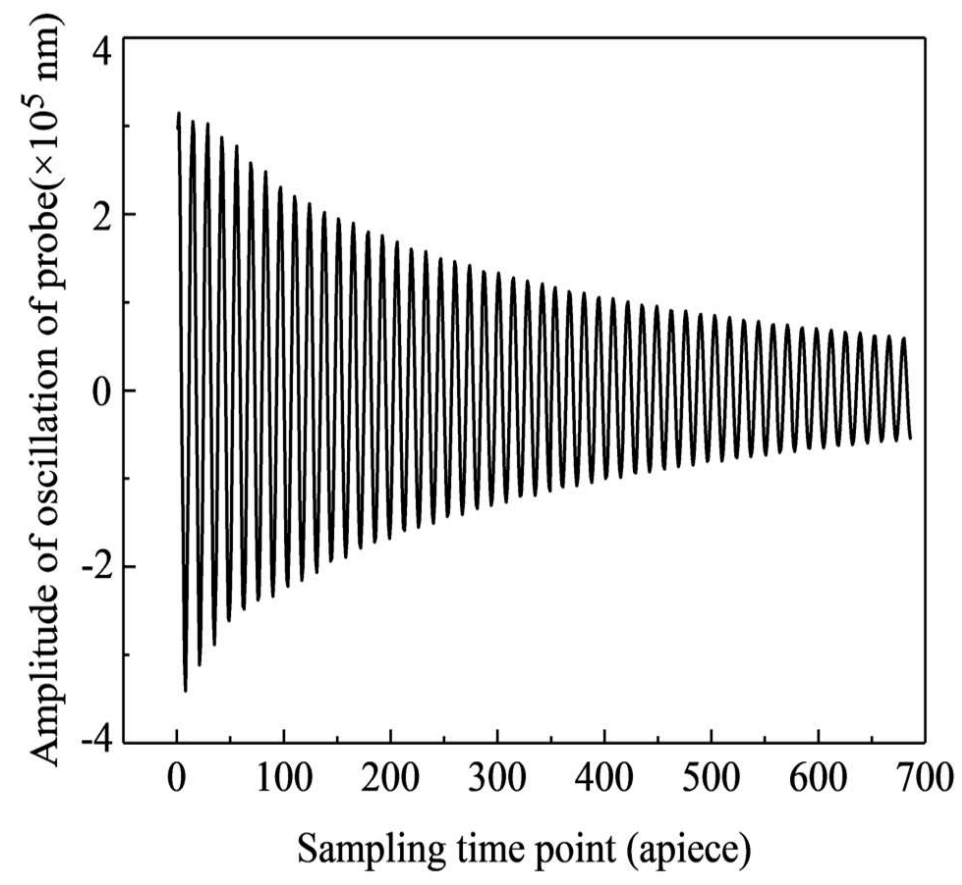

Fig. 16 Natural frequency measured by percussion method 


\section{Conclusions}

In this study, a model was established for a special probe of a 3D thread-measuring machine. The geometric parameters of each component of the probe were designed and optimized. The static and dynamic characteristics of the probe were analyzed by the finite element analysis method and verified experimentally. The research is summarized as follows:

(1) The "T"-shaped, ballpoint-pen shaped needle was selected according to the characteristics of the detection hole and thread, and a measuring rod was added. A parallel reed with high sensitivity was selected as the elastic element. The two groups of reeds were nested to make the structure compact, thereby ensuring accuracy.

(2) The optimum ball radius was determined as $0.02 \mathrm{~mm}$ according to Hertz's law. The flexural deformation of the measuring rod was analyzed to determine the appropriate length and diameter of the measuring rod, which were 100 and $4 \mathrm{~mm}$, respectively. The dimensions of the parallel reeds were determined according to the relationship between the maximum measuring force of the parallel reeds and the splint width, thickness, and length.

(3) ANSYS was used for the finite element analysis of the probe. The probe exhibited appropriate linearity, stiffness, and other static characteristics in addition to high natural frequency dynamic characteristics.

(4) The static characteristics of the probe were measured. The return error was $0.29 \mu \mathrm{m}$, and the repeatability error was $0.24 \mu \mathrm{m}$. The dynamic characteristics were tested using the percussion method, and the natural frequency was $180 \mathrm{~Hz}$. The static and dynamic characteristics were consistent with the finite element simulation results, which demonstrated the reliability of the simulation and the credibility of the evaluation of the probe characteristics.

This research explores the impact of several important dimensional parameters on the accuracy of the probe; however, it does not explore the impact of the overall structure of the probe on the accuracy, and does not explain the errors caused by the manufacturing and installation of the probe. The principal analysis method in this article may only be applicable to parallel reed probes. Further research will be conducted to verify the characteristics of the $3 \mathrm{D}$ thread measuring machine contact probe to confirm its application for different specifications of thread. This will be achieved through a more sophisticated approach of multi-factor interaction. In addition, for the geometry 
model of the probe, the error source will be analyzed, the mathematical model will be established, and the error compensation algorithm and the corresponding coordinate change matrix will be provided. The probe positioning error model will be examined, and the influence of measurement error caused by tilt angle will be analyzed emphatically.

\section{Acknowledgements}

The author gratefully acknowledges the support of professor Dong-sheng Li, Ultraprecision geometric measurement laboratory (China Jiliang University), and Precision measurement R\&D department (Chotest Technology Inc) in the framework of the program "Development and application of high precision three dimensional screw thread measuring instrument" (National Key R\&D Program of China, Project No.2018YFF01013102). We would like to thank Editage (www.editage.cn) for English language editing.

\section{References}

[1] Boby RA, Sonakar PS, Singaperumal M et al (2011) Identification of defects on highly reflective ring components and analysis using machine vision. Int J Adv Manuf Technol 52:217-233. https://doi.org/10.1007/s00170-010-2730-3

[2] Liu F (2006) Research on machine vision method of thread detection. Dissertation, Tianjin University. (in Chinese).

[3] Voulodimos A, Doulamis N, Doulamis A, Protopapadakis E (2018) Deep learning for computer vision: A brief review. Comput Intell Neurosci 2018:1-13. https://doi.org/10.1155/2018/7068349

[4] Peng EB, Zhang GT (2014) Image processing technology research of on-line thread processing. Adv Mater Res 908:555-558. https://doi.org/10.4028/www.scientific.net/AMR.908.555

[5] Gong Y, Zhang S (2011) High-resolution, high-speed three-dimensional shape measurement using projector defocusing. Opt Eng 50:023603. https://doi.org/10.1117/1.3534798

[6] Mian SH, Al-Ahmari A (2014) New developments in coordinate measuring 
machines for manufacturing industries. Int J Metrol Qual Eng 5:101. https://doi.org/10.1051/ijmqe/2014001

[7] Gong Y, Johnston RS, Melville CD, Seibel EJ (2015) Axial-stereo 3-D optical metrology for inner profile of Pipes using a scanning laser endoscope. Int $\mathrm{J}$ Optomechatronics 9:238-247. https://doi.org/10.1080/15599612.2015.1059535

[8] Gong Y, Seibel EJ (2017) Three-dimensional measurement of small inner surface profiles using feature-based 3-D panoramic registration. Opt Eng 56:014108. https://doi.org/10.1117/1.OE.56.1.014108

[9] Wakayama T, Takahashi Y, Ono Y et al (2018) Three-dimensional measurement of an inner surface profile using a supercontinuum beam. Appl Opt 57:5371-5379. https://doi.org/10.1364/AO.57.005371

[10]Cai CM (2013) Structural Design and Characteristics research of Micro-nanoprobe Based on the Principle of Micro-force Measurement. Dissertation, Anhui University of Science and Technology. (in Chinese).

[11] Fan KC, Chen Y, Wang W. Probe technologies for micro/nano measurements Hong Kong: 7th IEEE Conference on Nano technology, 2007. DOI: 10.1109/NANO.2007.4601349

[12]Li S (2018) Precision Analysis and Improvement of 3D Probe steering mechanism. Dissertation, Xi An University of Technology. (in Chinese).

[13]Li RJ, Fan KC, Huang QX et al (2016) A long-stroke 3D contact scanning probe for micro/nano coordinate measuring machine. Precis Eng 43:220-229. https://doi.org/10.1016/j.precisioneng.2015.08.001

[14]Ha SJ, Kim DW, Shin BC et al (2010) Assessment of design and mechanical characteristics of MEMS probe tip with fine pitch. J Korea Academia-Industrial Cooperation Society 11:1210-1215. https://doi.org/10.5762/KAIS.2010.11.4.1210

[15]Liang Q, Zhang D, Wang Y, Ge Y (2012) Development of a touch probe based on five-dimensional force/torque transducer for coordinate measuring machine (CMM). Robot Comput Integr Manuf 28:238-244. https://doi.org/10.1016/j.rcim.2011.09.004

[16]Elshamy M, Crosby WA, Elhadary M (2018) Crack detection of cantilever beam by natural frequency tracking using experimental and finite element analysis. Alex Eng J 57:3755-3766. https://doi.org/10.1016/j.aej.2018.10.002

[17]Chiu JT, Chang DY (2009) A new probe design combining finite element method and optimization used for vertical probe card in wafer probing. Precis Eng 33:395- 
401. https://doi.org/10.1016/j.precisioneng.2008.10.007

[18]Fan KC, Cheng F, Pan WT, Li R (2010) Analysis of the contact probe mechanism for micro-coordinate measuring machines. Optoelectron Instrum Data Process 46:340-346. https://doi.org/10.3103/S8756699010040060

[19]Oh YR, Kim YJ, Nam HS et al (2012) Design of vertical type probe tip using finite element analysis. Trans Korean Soc Mech Eng A 36:851-856. https://doi.org/10.3795/KSME-A.2012.36.8.851

[20]Meijaard JP, Brouwer DM, Jonker JB (2010) Analytical and experimental investigation of a parallel leaf spring guidance. Multibody Syst Dyn 23:77-97. https://doi.org/10.1007/s11044-009-9172-4

[21] Fan GZ, Li RJ (2008) Design and Verification of three-coordinate Measuring machine micro-nanoprobe. Measuring Technology 38:60-81. (in Chinese). 